\title{
Supplementary Materials for Water Plays a Co-catalytic Role in Epoxide Ring Opening Reaction in Aspartate Proteases: A QM/MM Study
}

\author{
Mohd Ahsan and Sanjib Senapati* \\ Department of Biotechnology and BJM School of Biosciences, \\ Indian Institute of Technology Madras, Chennai 600036, India. \\ Ph:+91-44-22574122, e-mail: sanjibs@iitm.ac.in
}

\section{List of Supplementary Materials}

Figures S1-S8

Tables ST1 

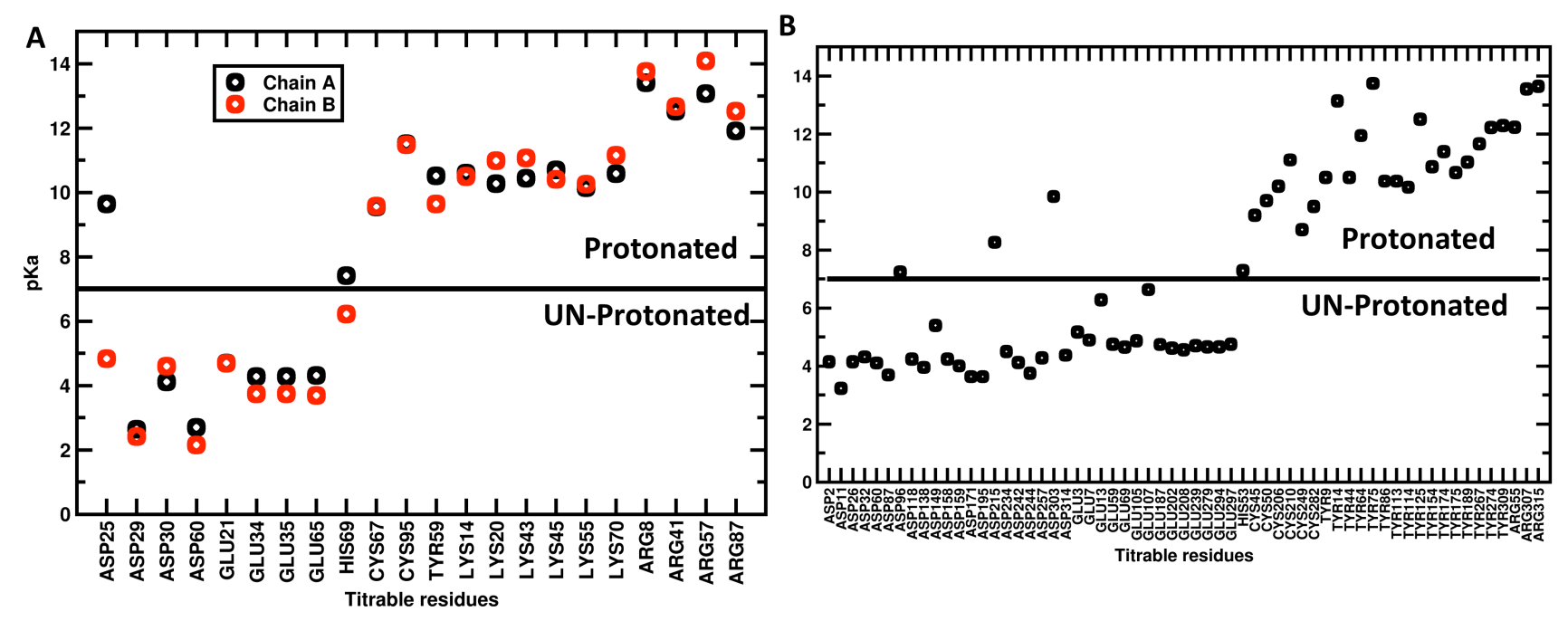

Figure S1: Estimated pKa values of the titratable residues of (A) HIV protease and (B) pepsin as obtained using PropKa 2.0 program. 
A

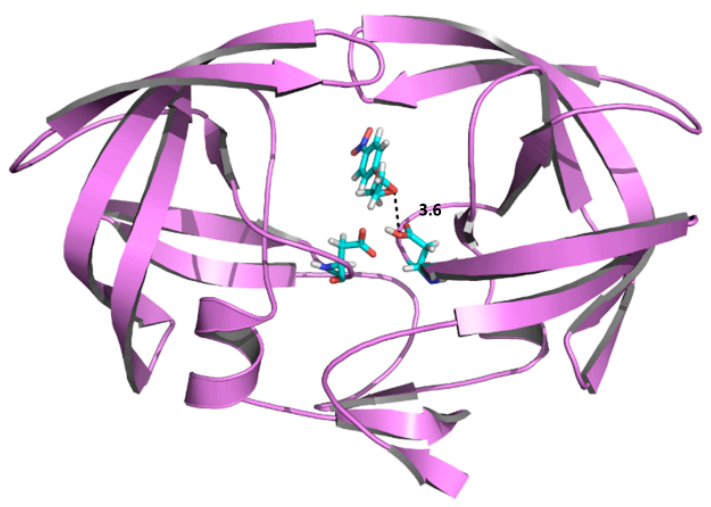

B

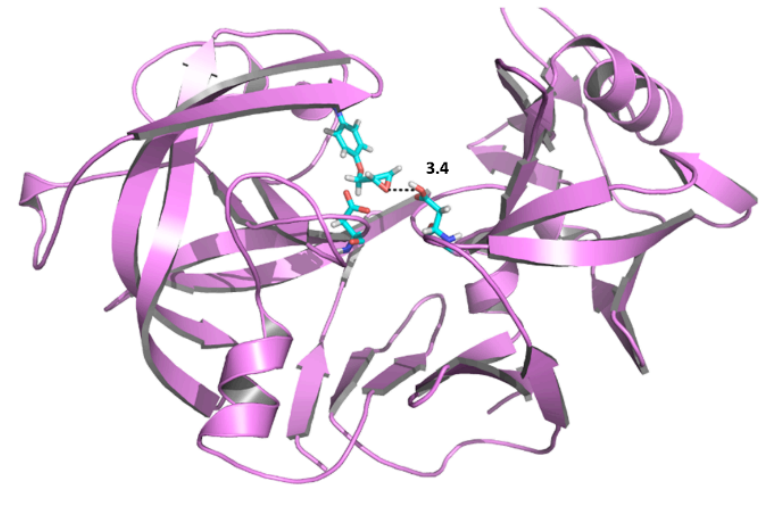

Figure S2: The lowest energy enzyme-EPNP docked complex for (A) HIV protease and (B) pepsin. Interatomic distance ( $\AA$ ) between EPNP and AspH is shown in black dashed line. 


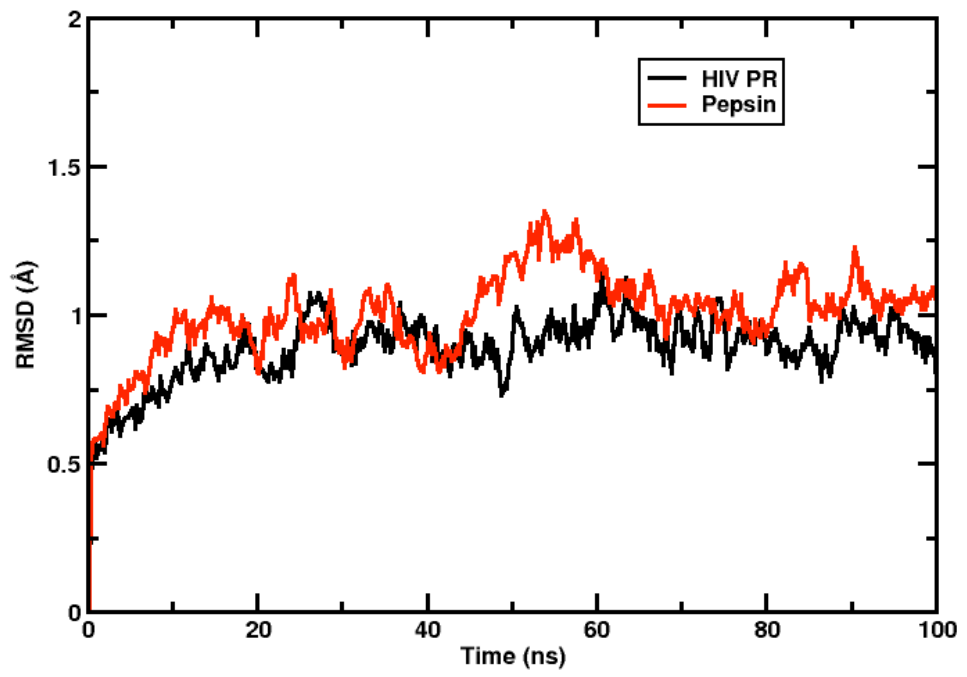

Figure S3: Time evolution of the Root Mean Squared Displacement (RMSD) of EPNP in the active site of HIV protease (black) and pepsin (red). From MD data, the epoxide ring was found to fit very well at $\sim 3 \AA$ from the catalytic $A s p H$, very similar to the binding of parent ligands pepstatin and amprenavir that were at $2.7 \AA$ and $2.8 \AA$ from $\mathrm{AspH}$ in the respective X-ray structures. 

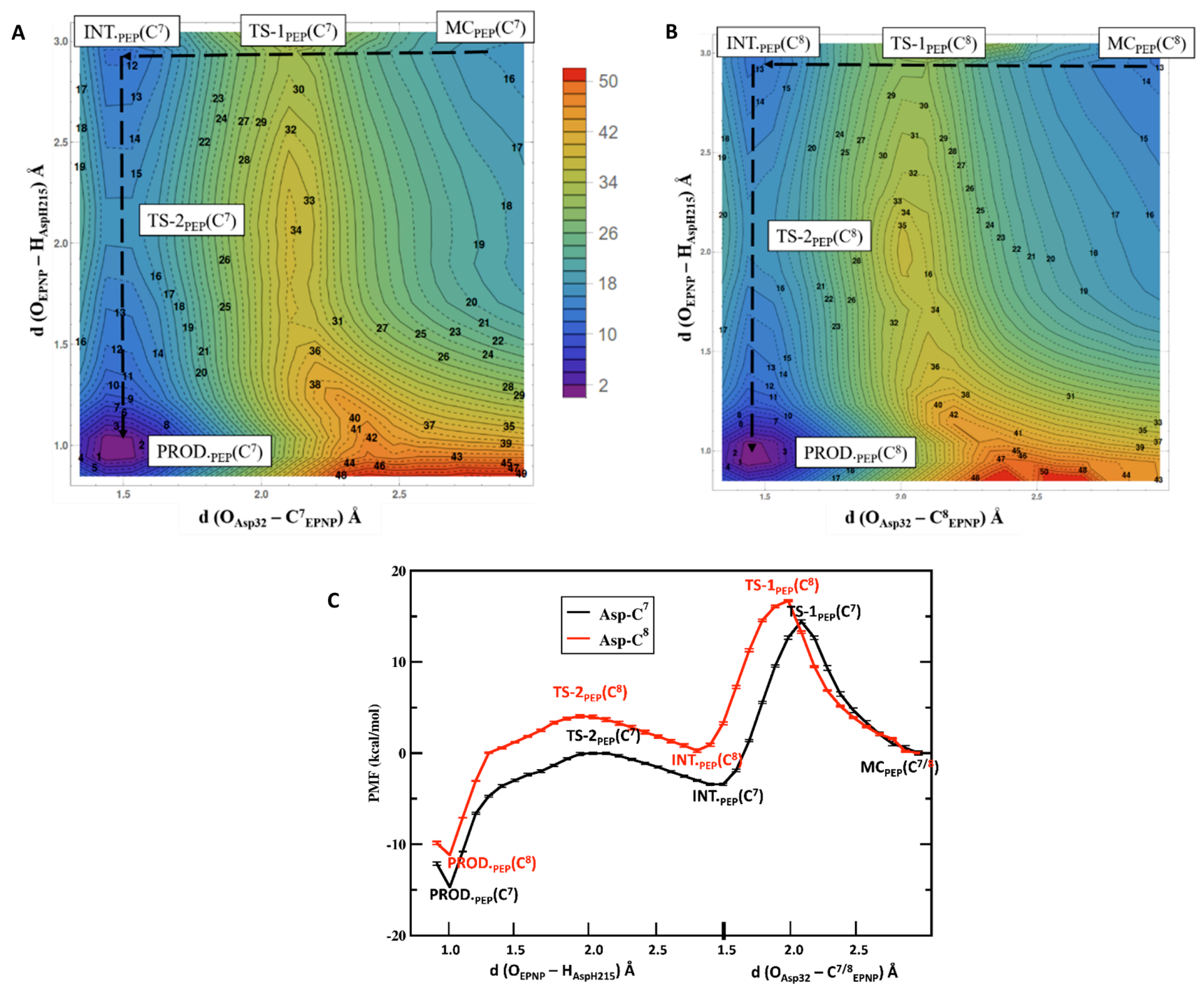

Figure S4: Alkylation of catalytic Asp32 in pepsin takes place through EPNP C ${ }^{7}$ carbon. 2D free energy profiles for the formation of enzyme-EPNP complex through the covalent linkage of (A) Asp32-C $\mathrm{C}^{7}$ (B) Asp32-C $\mathrm{C}^{8}$. The dashed lines indicate the lowest energy path from MC to Product. The iso-energetic lines represent energy in kcal.mol ${ }^{-1}$. (C) The $1 \mathrm{D}$ energy profiles of the transitions depicted in (A) and (B). Reaction coordinates are same as in (A) or (B). 


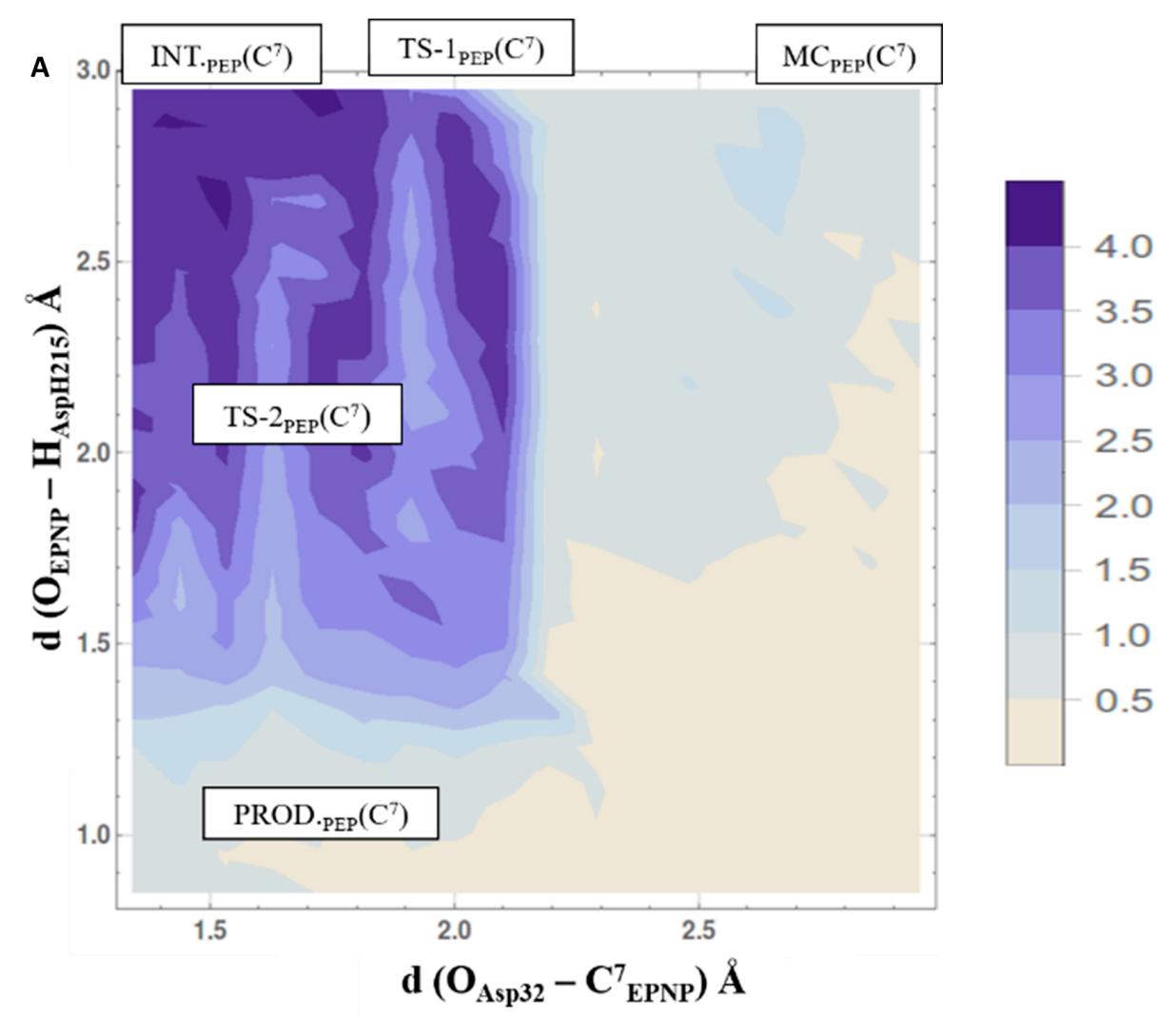

B

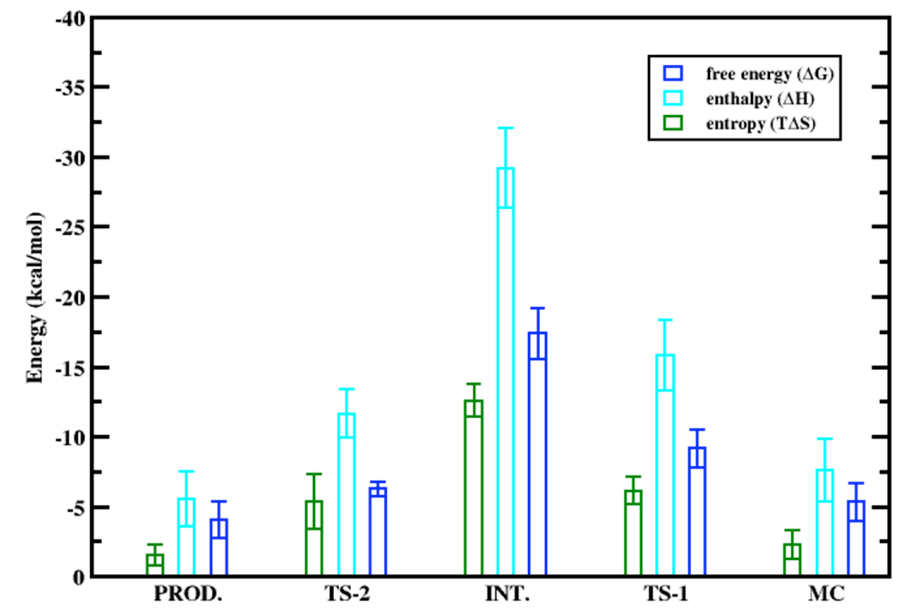

Figure S5: TS and intermediate are stabilized by low-barrier H-bonds. (A) Number of water in the active site that are H-bonded to epoxide ring over the reaction coordinates. Geometric criterion of H-bond is adopted: $\mathrm{O}_{\text {epo }}-\mathrm{O}_{\mathrm{w}}$ distance $<3.5 \AA$ and $\mathrm{O}_{\mathrm{w}}-\mathrm{H}_{\mathrm{w}}-\mathrm{O}_{\text {epo }}$ angle $>$ $135^{\circ}$. (B) Free energy $(\Delta \mathrm{G})$, enthalpy $(\Delta \mathrm{H})$, and entropy $(\mathrm{T} \Delta \mathrm{S})$ of epoxide-water adduct formation at different states. 

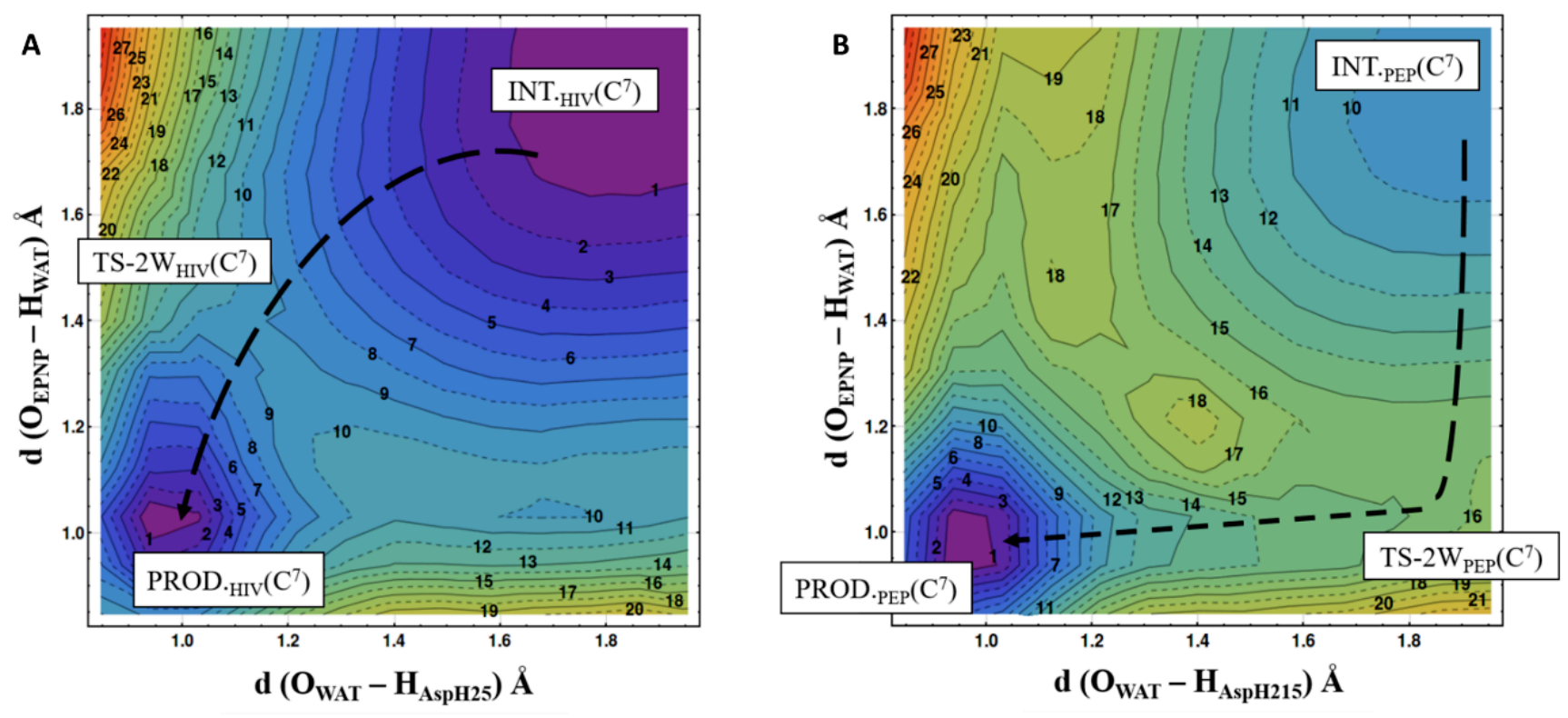

Figure S6: The 2D free energy profile for the water mediated proton transfer, i.e. water takes up the proton from $\mathrm{AspH}$ and then transfers to $\mathrm{O}_{\mathrm{EPNP}}$ in the intermediate. Results are shown for (A) HIV protease (B) pepsin. 

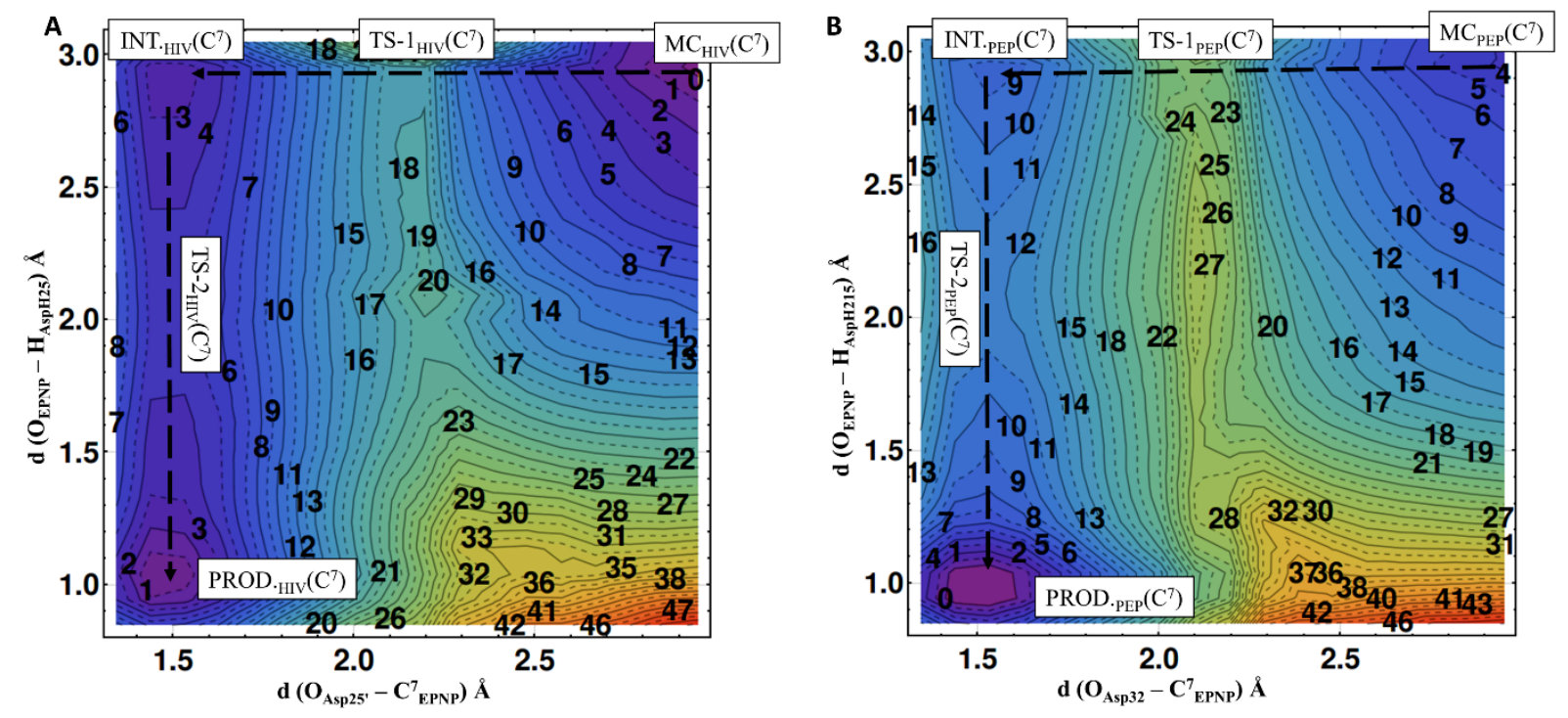

Figure S7: Corrected 2D free energy profile at M06-2X/6-31+G(d,p)/MM level of accuracy for (A) HIV protease (B) pepsin. 

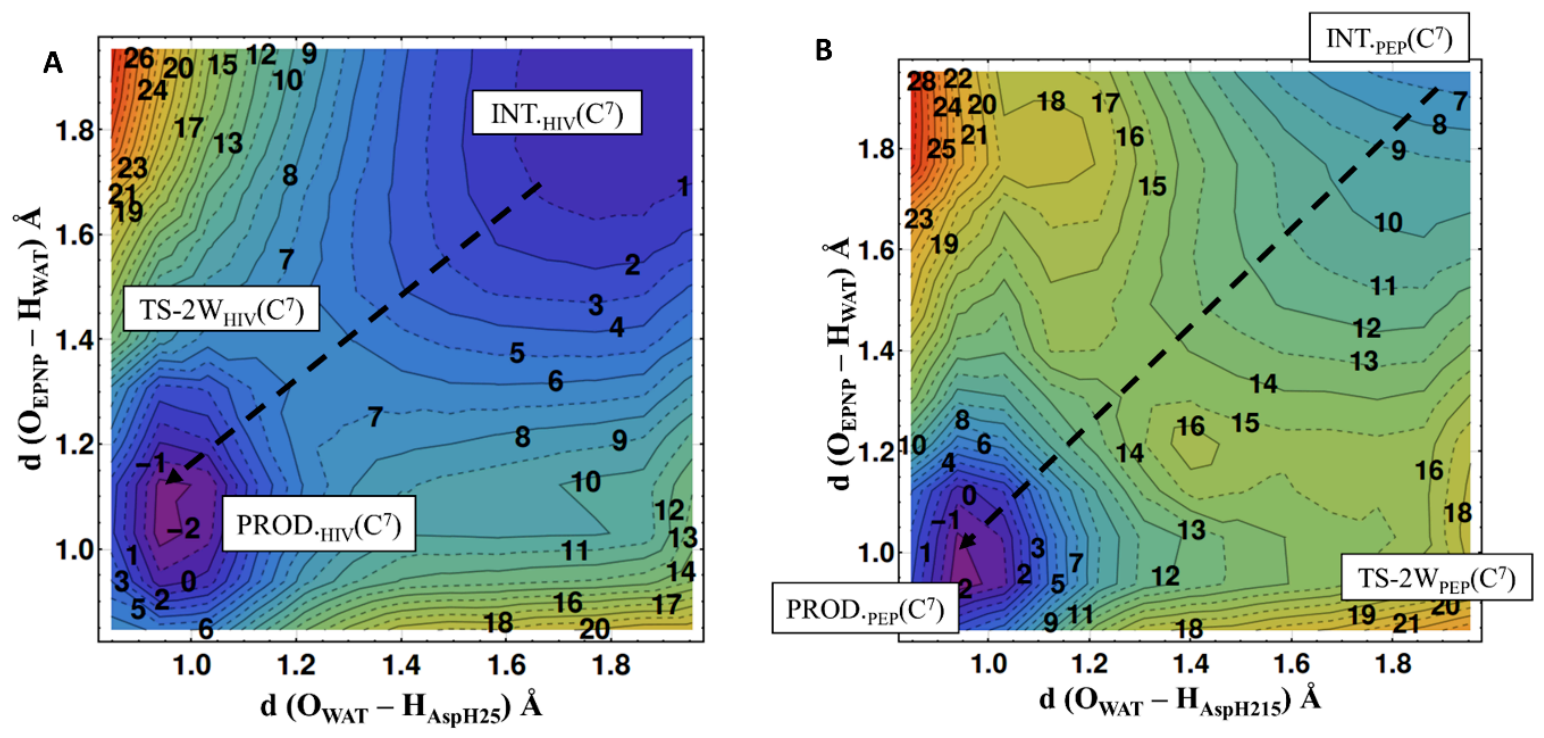

Figure S8: Corrected 2D free energy profile at M06-2X/6-31+G(d,p)/MM level of accuracy for the water mediated proton transfer. Results are shown for (A) HIV protease (B) pepsin. 
Table ST1: Atomic charge (in a.u.) computed using CHelpG method for the bounded (protein) and unbounded (water) structures of EPNP at DFT(M06-2X/6-31+G(d,p)) level of theory.

\begin{tabular}{|c|l|l|l|}
\hline Atom Name & \multicolumn{1}{|c|}{$\begin{array}{c}\text { EPNP in } \\
\text { Water }\end{array}$} & $\begin{array}{c}\text { EPNP complex } \\
\text { with HIVPR }\end{array}$ & $\begin{array}{c}\text { EPNP complex } \\
\text { with pepsin }\end{array}$ \\
\hline $\mathbf{O 2}_{\text {EPNP }}$ & $-0.404 \pm 0.018$ & $-0.362 \pm 0.031$ & $-0.396 \pm 0.096$ \\
\hline $\mathbf{N}_{\text {EPNP }}$ & $0.647 \pm 0.051$ & $0.599 \pm 0.020$ & $0.624 \pm 0.023$ \\
\hline $\mathbf{O 3}_{\text {EPNP }}$ & $-0.405 \pm 0.024$ & $-0.450 \pm 0.040$ & $-0.440 \pm 0.031$ \\
\hline $\mathbf{C 3}_{\text {EPNP }}$ & $-0.044 \pm 0.109$ & $-0.054 \pm 0.013$ & $-0.093 \pm 0.014$ \\
\hline $\mathbf{C 4}_{\text {EPNP }}$ & $0.090 \pm 0.072$ & $0.107 \pm 0.022$ & $0.077 \pm 0.023$ \\
\hline $\mathbf{C 5}_{\text {EPNP }}$ & $-0.109 \pm 0.020$ & $-0.052 \pm 0.050$ & $-0.149 \pm 0.022$ \\
\hline $\mathbf{C}_{\text {EPNP }}$ & $0.076 \pm 0.056$ & $0.049 \pm 0.040$ & $0.136 \pm 0.029$ \\
\hline $\mathbf{C 1}_{\text {EPNP }}$ & $-0.121 \pm 0.014$ & $-0.131 \pm 0.046$ & $-0.139 \pm 0.017$ \\
\hline $\mathbf{C}_{\text {EPNP }}$ & $0.448 \pm 0.035$ & $0.402 \pm 0.061$ & $0.463 \pm 0.040$ \\
\hline $\mathbf{O}_{\text {EPNP }}$ & $-0.433 \pm 0.030$ & $-0.327 \pm 0.026$ & $-0.382 \pm 0.032$ \\
\hline $\mathbf{C 6} \mathbf{E P N P}_{\text {EPN }}$ & $0.333 \pm 0.026$ & $0.236 \pm 0.064$ & $0.276 \pm 0.054$ \\
\hline $\mathbf{C} 7_{\text {EPNP }}$ & $\mathbf{0 . 1 0 3} \pm \mathbf{0 . 0 2 7}$ & $\mathbf{0 . 2 0 5} \pm \mathbf{0 . 0 7 8}$ & $\mathbf{0 . 2 7 8} \pm \mathbf{0 . 0 3 8}$ \\
\hline $\mathbf{C 8}_{\text {EPNP }}$ & $\mathbf{0 . 1 5 8} \pm \mathbf{0 . 0 2 5}$ & $\mathbf{0 . 0 3 0} \pm \mathbf{0 . 0 1 6}$ & $\mathbf{0 . 2 1 6} \pm \mathbf{0 . 0 2 9}$ \\
\hline $\mathbf{O 1}_{\text {EPNP }}$ & $-0.311 \pm 0.012$ & $-0.279 \pm 0.014$ & $-0.445 \pm 0.014$ \\
\hline
\end{tabular}

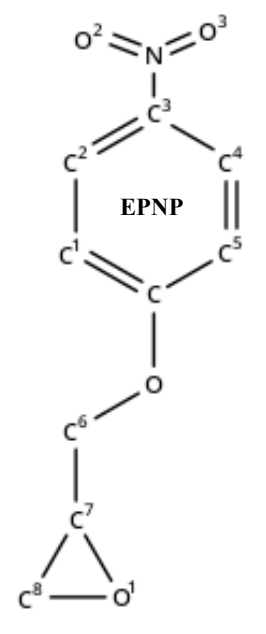

